\title{
miR-380-5p facilitates NRF2 and attenuates cerebral ischemia/reperfusion injury-induced neuronal cell death by directly targeting BACH1
}

https://doi.org/10.1515/tnsci-2020-0172

received November 25, 2020; accepted April 19, 2021

\begin{abstract}
Background - This study aimed to explore the role of miR-380-5p in cerebral ischemia/reperfusion (CIR) injuryinduced neuronal cell death and the potential signaling pathway involved.

Methodology - Human neuroblastoma cell line SH-SY5Y cells were used in this study. Oxygen and glucose deprivation/reperfusion (OGD/R) model was used to mimic ischemia/reperfusion injury. CCK- 8 assay and flow cytometry were used to examine cell survival. Quantitative real time PCR (RT-qPCR) assay and Western blotting were used to measure the change of RNA and protein expression, respectively. TargetScan and Luciferase assay was used to confirm the target of miR-380-5p. Malondialdehyde (MDA) superoxide dismutase (SOD) and glutathione peroxidase (GSHPx) were measured using commercial kits.

Results - miR-380-5p was downregulated in SH-SY5Y cells after OGD/R. Cell viability was increased by miR380-5p, while cell apoptosis was reduced by miR-380$5 p$ mimics. MDA was reduced by miR-380-5p mimics, while SOD and GSHPx were increased by miR-380-5p. Results of TargetScan and luciferase assay have showed that BACH1 is the direct target of miR-380-5p. Expression of NRF2 was upregulated after OGD/R, but was not affected by miR-380-5p. mRNA expression of HO-1 and NQO1 and ARE activity were increased by miR-380-5p. Overexpression of BACH1 reversed the antioxidant and neuroprotective effects of miR-380-5p.
\end{abstract}

\footnotetext{
* Corresponding author: Min Xu, Department of Neurosurgery, Kunshan Hospital of Traditional Chinese Medicine, Kunshan Affiliated Hospital of Nanjing University of Chinese Medicine, No. 189 Chaoyang Road, Kunshan City, Jiangsu Province, 215300, China, e-mail: minxu06251505@163.com, tel: + 86-0512-5731-0000 Yibiao Wang: Department of Neurosurgery, Hainan Affiliated Hospital of Hainan Medical University, Haikou City, Hainan Province, 570311, China
}

Conclusion - miR-380-5p inhibited cell death induced by CIR injury through target BACH1 which also facilitated the activation of NRF2, indicating the antioxidant and neuroprotective effects of miR-380-5p.

Keywords: miR-380-5p, NRF2, cerebral ischemia/reperfusion injury, neuronal cell death, BACH1

\section{Introduction}

Approximately $80 \%$ of strokes were caused by cerebral ischemia, which is the most common atherosclerotic disease in the cerebral blood vessels [1,2]. Cerebral ischemia/ reperfusion (CIR) injury could result in serious dysfunction of brain with high risk of disability and mortality [3]. If the cerebral ischemia was not reversed within a short time, neuronal cell death would be observed around the affected vessels, termed infarction [5]. So far, there are no effective treatment for CIR injury. Thus, the therapeutic strategies of CIR injury aim to prevent neuronal cell death, which will reduce the infarct size [4].

During cerebral ischemia, the blood supply to brain was reduced, which led to the reduction of oxygen and glucose supply to the affected brain tissue [6]. Aerobic metabolism promoted the abundance of reactive oxygen species (ROS), a type of free radical, which contributed to cell necrosis and death lipid peroxidation [7]. In normal condition, ROS can be scavenged by superoxide dismutase (SOD), the most crucial antioxidant enzyme in brain [8]. SOD can convert $\mathrm{O}_{2}^{-}$into $\mathrm{H}_{2} \mathrm{O}_{2}$, and further into $\mathrm{H}_{2} \mathrm{O}$ and $\mathrm{O}_{2}$ by glutathione peroxidase (GSHPx) [7,9]. Therefore, increase of SOD and GSHPx activity could prevent cell death during CIR injury. In addition to SOD and GSHPx, nuclear factor erythroid 2-related factor 2 (NRF2) also involved into the transcription of antioxidant genes and activation of NRF2 and its target genes could prevent CIR injury in brain [10].

As a class of small noncoding RNAs, miRNAs participate in many physiopathological processes through inducing translational repression of their target mRNAs [11]. 
Dysregulation of miRNAs after CIR injury was observed using miRNA-profiling technique, suggesting that miRNAs engaged in the response to CIR injury [12]. Eight miRNAs have been upregulated after $3 \mathrm{~h}$ of reperfusion and, among them, miR200b, miR-200c, and miR-429 have demonstrated their neuroprotective effects through targeting prolyl hydroxylase 2 [13]. The goal of this study is to figure out the dysregulation of miR-380-5p and its role in CIR injury-induced neuronal cell death, providing new insight for the treatment of CIR injury.

\section{Methods}

\subsection{Cell culture and treatment}

Human neuroblastoma cell line SH-SY5Y cells were obtained from American Type Culture Collection (ATCC, USA) and cultured into Dulbecco's Modified Eagle's Medium (DMEM) that contained 1\% penicillin/streptomycin (Invitrogen, USA) and 10\% fetal bovine serum (FBS, Gibco, USA) at $37^{\circ} \mathrm{C}$ with $95 \%$ air and $5 \% \mathrm{CO}_{2}$ (normal condition) in a humidified incubator.

Oxygen and glucose deprivation/reperfusion (OGD/R) model was used to mimic ischemia/reperfusion injury in this study. Briefly, SH-SY5Y cells were cultured with glucose-free DMEM with $95 \% \mathrm{~N}_{2}$ and $5 \% \mathrm{CO}_{2}$ (anaerobic condition) for $6 \mathrm{~h}$ and then cultured in normal condition for another $24 \mathrm{~h}$ to induce reoxygenation.

miR-380-5p mimics, miR-380-5p inhibitors, and their negative controls were obtained from Guangzhou RiboBio Co., LTD (RiboBio, China). BACH1-overexpression vectors and its control vectors were also purchased from RiboBio. $1 \times 10^{5}$ cells per well of SH-SY5H cells were incubated in the 6 well plates. Before $48 \mathrm{~h}$ of $\mathrm{OGD} / \mathrm{R}$, cell transfection or co-transfection was conducted using lipofectamine 2000 according to the manufacturer's instructions.

Ethical approval: The conducted research is not related to either human or animals use.

\subsection{Examination of cell viability and apoptosis}

Cell viability was detected using CCK-8 kit (Sigma-Aldrich, USA). After treated as described above, SH-SY5Y cells were seeded in the 96-well plate at the density of $10^{4}$ cells per well and cultured at $37^{\circ} \mathrm{C}$ overnight. The medium was removed, and the cells were washed with PBS. $10 \mu \mathrm{L}$ of CCK- 8 reagent was added to each well and incubated at $37^{\circ} \mathrm{C}$ for $2 \mathrm{~h}$. The optical density value at $450 \mathrm{~nm}$ was measured and recorded using a Bio-Rad microplate reader.

Cell apoptosis was measured using flow cytometry. After treated as described above, SH-SY5Y cells were resuspended and stained using Annexin V-FITC Apoptosis Detection Kit (Sigma-Aldrich). Ten thousand cells were collected using flow cytometry (FACScan, USA) and the results were analyzed using FlowJo ${ }^{\mathrm{TM}}$ v10.6.1 (FlowJo, USA).

\subsection{Measurement of malondialdehyde (MDA), SOD, GSHPx, and antioxidant response element (ARE)}

Change of MDA, SDO, GSHPx, and ARE was measured using the commercial kits, Lipid Peroxidation (MDA) Assay Kit (Sigma-Aldrich), Superoxide Dismutase Activity Assay Kit (Abcam, United Kingdom), Glutathione Peroxidase Assay Kit (Cayman Chemicals, USA), and ARE Reporter Kit (Bioscience, USA), respectively, according to the manufacturer's protocols.

\subsection{Luciferase assay}

The $3^{\prime}$ untranslated region (3'-UTR) of BACH1 and its mutant sequence (GeneCopoeia, USA) were inserted into the pGL3 vector (Promega, USA) to form luciferase reporter plasmids, BACH1-WT and BACH1-MUT, respectively. miR380-5p mimics, miR-380-5p inhibitors or their negative controls, were then co-transfected with BACH1-WT and BACH1-MUT, respectively. The change of luciferase activity was measured using the Promega Dual-Luciferase Reporter Assay System.

\subsection{RNA extract and quantitative real time PCR (RT-qPCR) assay}

Total RNA was extracted using PureLink ${ }^{\mathrm{TM}}$ RNA Mini Kit (Thermo Fisher, USA). 10 ng of RNA was used to reverse 
transcription to synthesize cDNA using QuantiTect Reverse Transcription Kit (QIAGEN, Germany), and RT-qPCR was performed using QIAGEN OneStep RT-PCR Kit (QIAGEN). RT-qPCR of miR-380-5p was performed using miScript SYBR $^{\circledR}$ Green PCR Kit (QIAGEN). Relative expression of RNAs was quantified using $2^{-\Delta \Delta \mathrm{CT}}$ method [14]. The primers used in this study were: U6 forward 5'-CTCGCTTCG GCAGCACA- $3^{\prime}$ and reverse $5^{\prime}$-AACGCTTCACGAATTTG CGT-3'; HO-1, forward 5'-ATGACACCAAGGACCAGAGC-3' and reverse $5^{\prime}$-TGTAAGGACCCATCGGAGA-3'; NQ01, forward $5^{\prime}$-CTCGCCTCATGCGTTTTTG- ${ }^{\prime}$ and reverse $5^{\prime}$-CCCC TAATCTGACCTCGTTCAT- $3^{\prime}$; $\beta$-actin forward, $5^{\prime}$-AGCCTCG CCTTTGCCGA- ${ }^{\prime}$ and reverse, 5'-CTGGTGCCTGGGGCG-3'.

\subsection{Western blotting}

The treated SH-SY5Y cells were lysed to extract the proteins using RIPA lysis and extraction buffer (Thermo Fisher). Nuclear proteins were extracted using Cytoplasmic And Nuclear Protein Extraction Kit (BosterBio, USA). $5 \mu$ g of total protein was loaded into $8 \%$ of sodium dodecyl sulphate-polyacrylamide gel and separated by electrophoresis. The separated protein was transferred from the gels to the PVDF membranes and the PVDF membranes were blocked by $5 \%$ of fat-free milk for $1 \mathrm{~h}$ at room temperature. The proper primary antibodies were used to probe the target proteins overnight at $4^{\circ} \mathrm{C}$ followed by incubating the membrane with proper secondary antibodies (ThermoFisher) for $2 \mathrm{~h}$ at room temperature. The strength of protein signal was detected using SignalFire ${ }^{\mathrm{TM}}$ ECL Reagent (CST, USA). The primary antibodies used in this study were: BACH1 (sc-271211, 1:1,000 dilution), NRF2 (ab137550, 1:1,000 dilution), Lamin B2 (ab233530, 1:800 dilution), and $\beta$-actin (ab8227, 1:5,000 dilution).

\subsection{Statistical analysis}

All statistical analyses were performed using SPSS v19.0 (IBM, USA). All experiment data were presented as mean \pm standard error of mean (SEM). Student's $t$ test and oneway ANOVA were used to compare the difference between two groups and multiple groups, respectively. $p$ value less than 0.05 was considered statistically significant difference.

\section{Results}

\subsection{Upregulation of miR-380-5p promoted cell survival and inhibited cell apoptosis induced by $\mathrm{OGD} / \mathrm{R}$}

Expression of miR-380-5p was significantly downregulated at 4,8 , and $12 \mathrm{~h}$ after OGD/R in SH-SY5Y cells (Figure 1). Cell viability was greatly reduced after OGD/ $\mathrm{R}$ (Figure 2a). The reduction of cell viability was further enhanced by inhibition of miR-380-5p and was reversed by overexpression of miR-380-5p (Figure 2a). The number of apoptotic cells was significantly increased after OGD/R (Figure 2b). Cell apoptosis was increased by miR-380-5p inhibitors and reduced by miR-380-5p mimics after OGD/R (Figure 2b).

\subsection{Overexpression of miR-380-5p repressed the oxidative stress after OGD/R}

After OGD/R, MDA level was increased in SH-SY5Y cells (Figure 3a). MDA level was further increased after OGD/R in cells transfected with miR-380-5p inhibitors compared with control group, while the OGD/R-induced increase of MDA was inhibited in cells transfected with miR380-5p mimics compared with control group (Figure 3a). After OGD/R, SOD was significantly reduced in SH-SY5Y cells (Figure $3 b$ ). The reduction of SOD induced by OGD/R was promoted by miR-380-5p inhibitors and prevented by miR-380-5p mimics (Figure 3b). Similarly, GSHPx was significantly reduced after OGD/R in SH-SY5Y

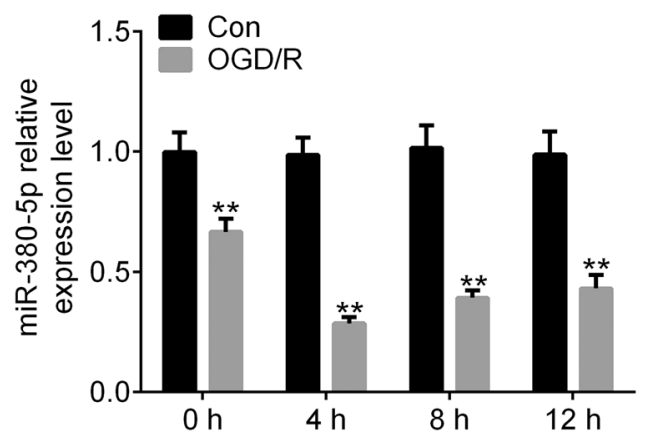

Figure 1: Expression of miR-380-5p was downregulated by $O G D / R$. ${ }^{\star *} p<0.01$ vs Con. Con: Control; OGD/R: oxygen and glucose deprivation/reperfusion. 
(a)

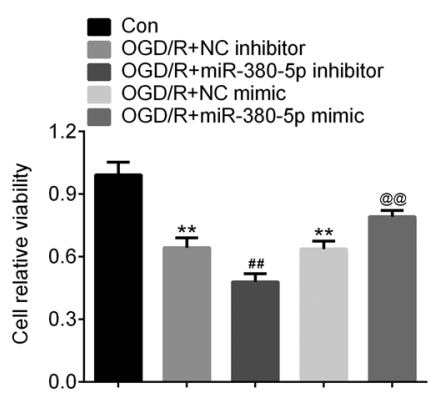

(b)

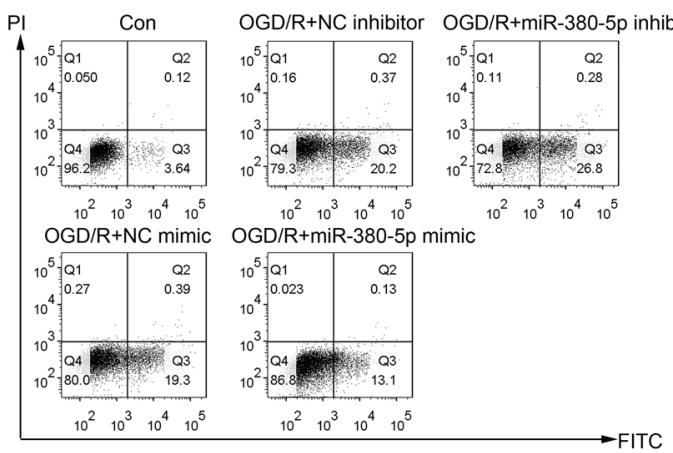

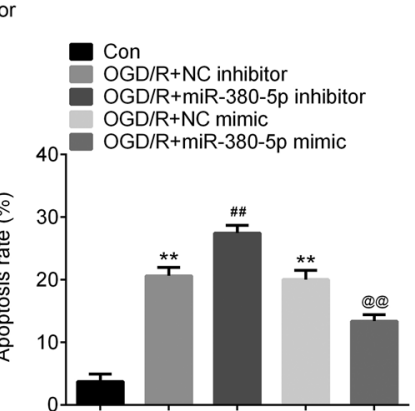

Figure 2: Upregulation of miR-380-5p promoted cell survival and inhibited cell apoptosis induced by OGD/R. (a) miR-380-5p prevented the reduction of cell viability induced by OGD/R. (b) miR-380-5p inhibited cell apoptosis induced by OGD/R. ** $p<0.01$ vs Con; ${ }^{\# \#} p<0.01$ vs NC inhibitor; $@^{@} p<0.01$ vs NC mimics. NC: negative inhibitor.

(a)

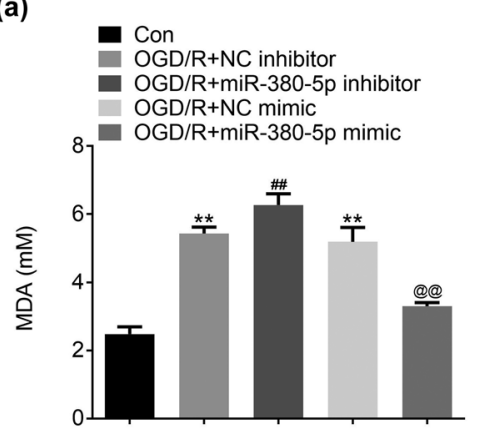

(b)

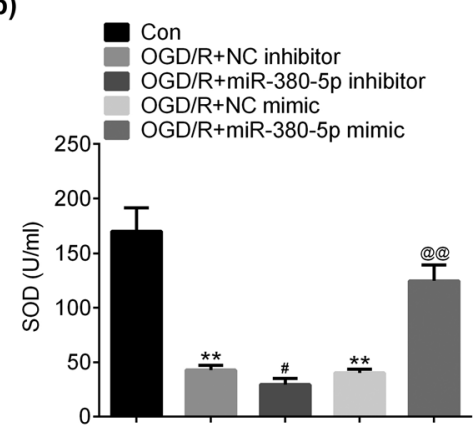

(c)

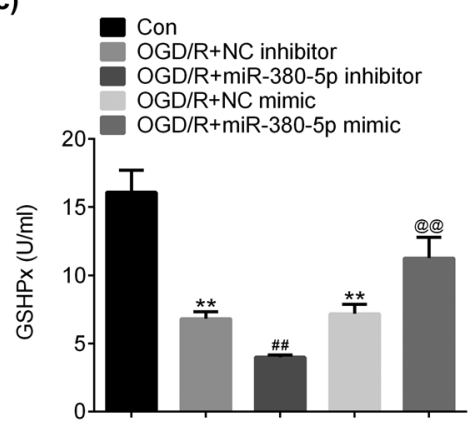

Figure 3: Overexpression of miR-380-5p repressed the oxidative stress after OGD/R. (a) miR-380-5p inhibited OGD/R-induced increase of MDA. (b) miR-380-5p inhibited OGD/R-induced reduction of SOD. (c) miR-380-5p inhibited OGD/R-induced reduction of GSHPx. ${ }^{\star *} p<0.01$ vs Con; ${ }^{\# \#} p<0.01$ vs NC inhibitor; ${ }^{@} p<0.01$ vs NC mimics. MDA: malondialdehyde; SOD: superoxide dismutase; GSHPx: glutathione peroxidase.

cells (Figure 3c). The reduction of GSHPx induced by OGD/R was promoted by miR-380-5p inhibitors and prevented by miR-380-5p mimics (Figure 3c).

\section{3 miR-380-5p directly targeted the $3^{\prime}$-UTR of BACH1}

TargetScan (www.targetscan.org) was used to predict the potential target of miR-380-5p. The results demonstrated that there was a complementary sequence between 3'-UTR of BACH1 and miR-380-5p (Figure 4a). Luciferase assay showed that the luciferase activity was increased by miR-380-5p inhibitors and reduced by miR-308-5p in cell co-transfected with BACH1-WT, while the luciferase activity did not show any significant change in cells cotransfected with BACH1-MUT (Figure 4b). After OGD/R, both BACH1 and NRF2 expressions were upregulated in SH-SY5Y cells (Figure 4c). The expression of BACH1 was upregulated by miR-380-5p inhibitors and downregulated by miR-380-5p mimics compared with negative control groups (Figure 4c). The expression of NRF2 was not affected by either miR-380-5p inhibitors or miR-380-5p mimics (Figure 4c). In cell nucleus, overexpression of NRF2 induced by OGD/R was downregulated by miR380-5p inhibitors and upregulated by miR-380-5p mimics (Figure 4d). The relative activity of ARE was increased after OGD/R (Figure 4e). The increased activity of ARE was inhibited by miR-380-5p inhibitors and induced by miR-380-5p mimics (Figure 4e). The mRNA level of HO-1 and NQO1 was upregulated after OGD/R (Figure 4f). Inhibition of miR-380-5p downregulated HO-1 and NQO1 mRNA expression and overexpression of miR-380-5p upregulated HO-1 and NQO1 mRNA expression (Figure 4f) 
(a)

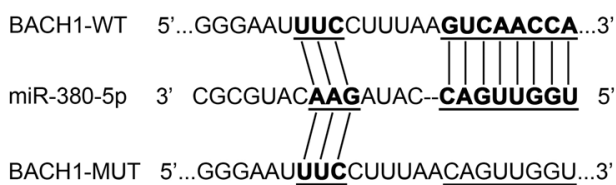

(b)

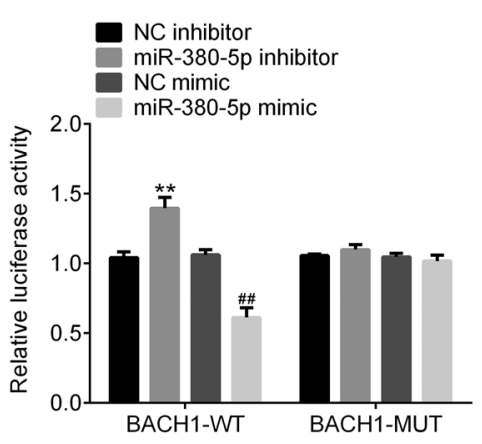

(c)

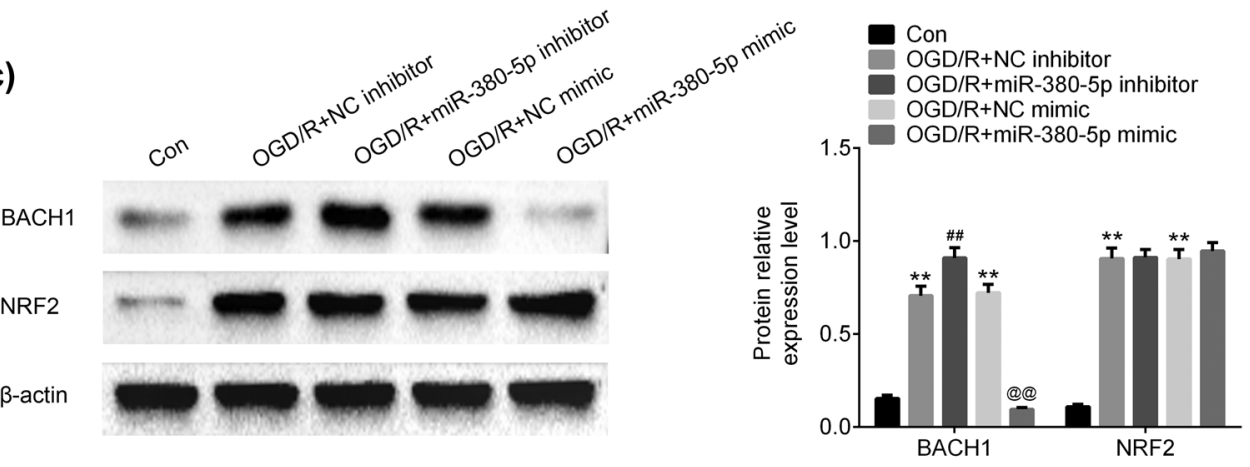

(d)

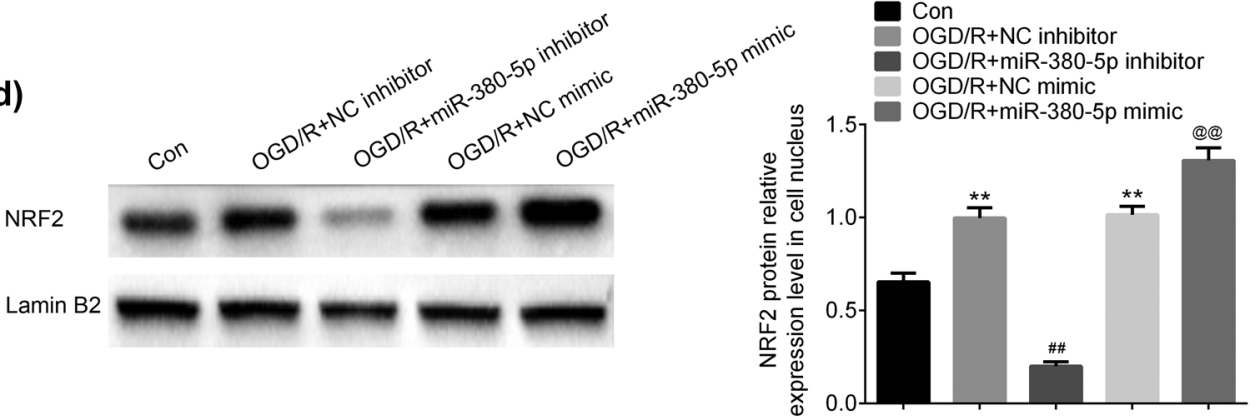

(e)

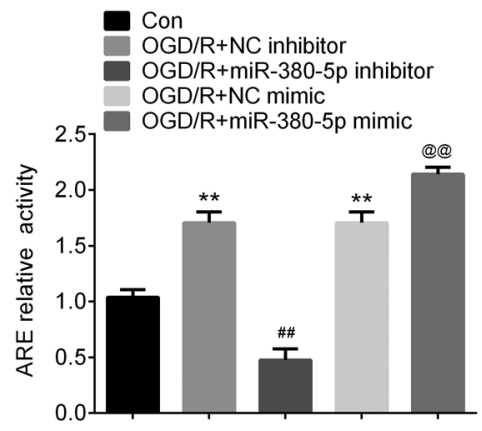

(f)

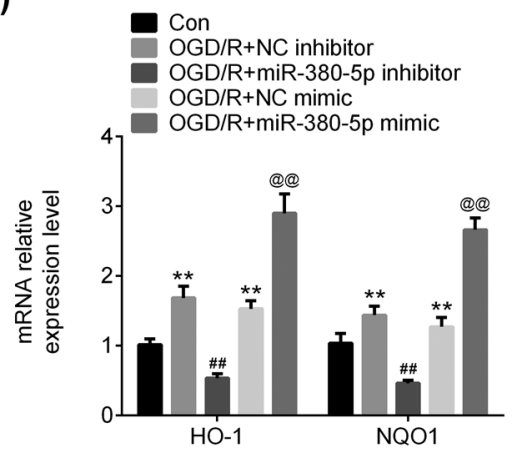

Figure 4: miR-380-5p directly targeted the $3^{\prime}$-UTR of BACH1. (a) A complementary sequence was observed between $3^{\prime}$-UTR of BACH1 and miR-380-5p. (b) Luciferase activity was increased by miR-380-5p inhibitors and decreased by miR-380-5p mimics in BACH1-WT group. (c) Expression of BACH1 was downregulated by miR-380-5p mimics, while expression of NRF2 did not show any significant difference. (d) Expression of BACH1 was upregulated by miR-380-5p mimics in cell nucleus. (e) miR-380-5p promoted the increase of ARE activity induced by OGD/R. (f) mRNA expression of HO-1 and NQ01 was upregulated by miR-380-5p after OGD/R. ${ }^{\star *} p<0.01$ vs Con; ${ }^{\# \#} p<0.01$ vs NC inhibitor; ${ }^{@} p<0.01$ vs NC mimics. 
(a)

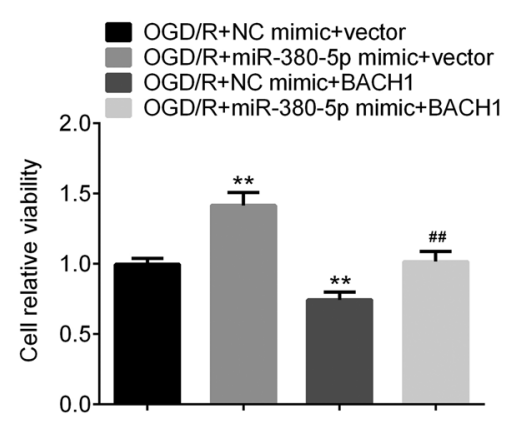

(c)

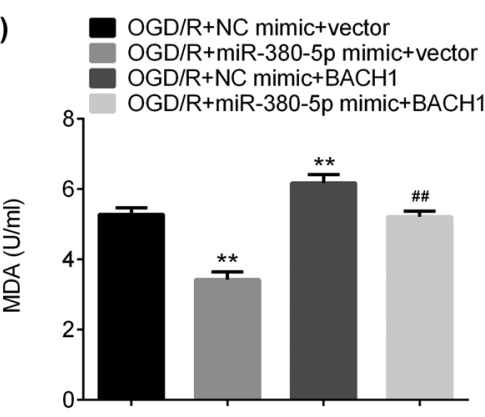

(f)

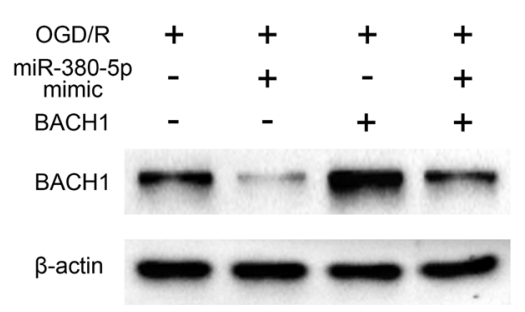

(h)

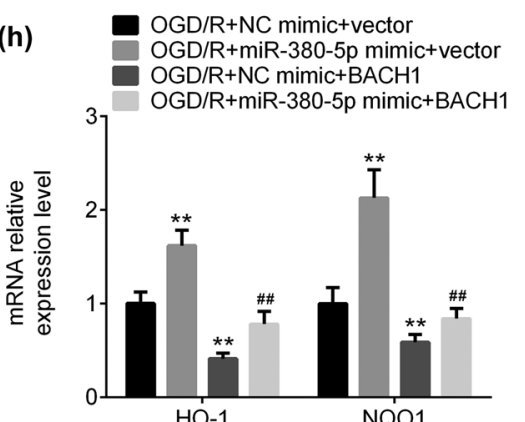

(b) PI OGD/R+NC mimic OGD/R+miR-380-5p mimic
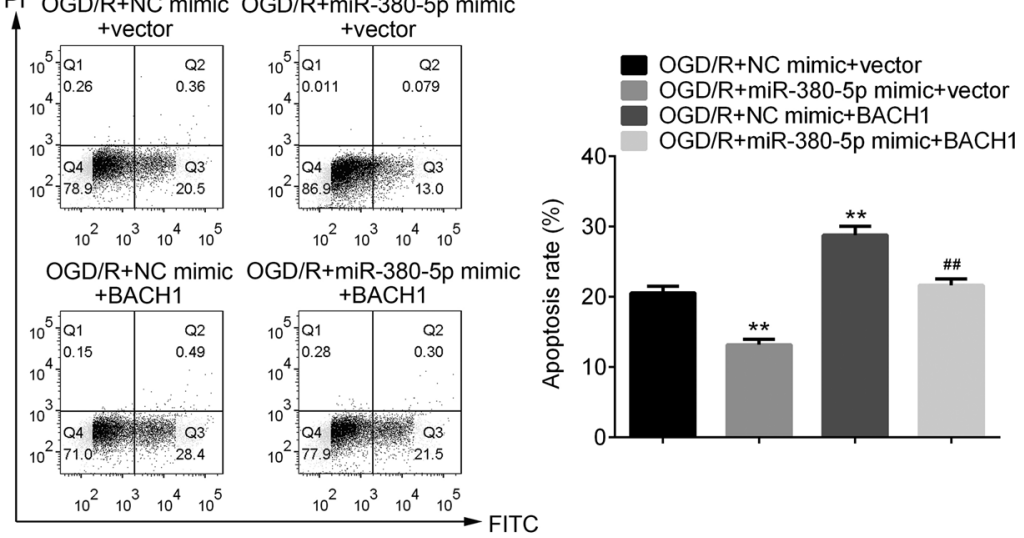

(d)

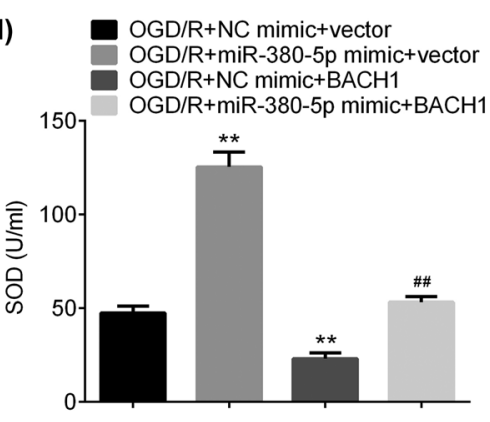

(e)
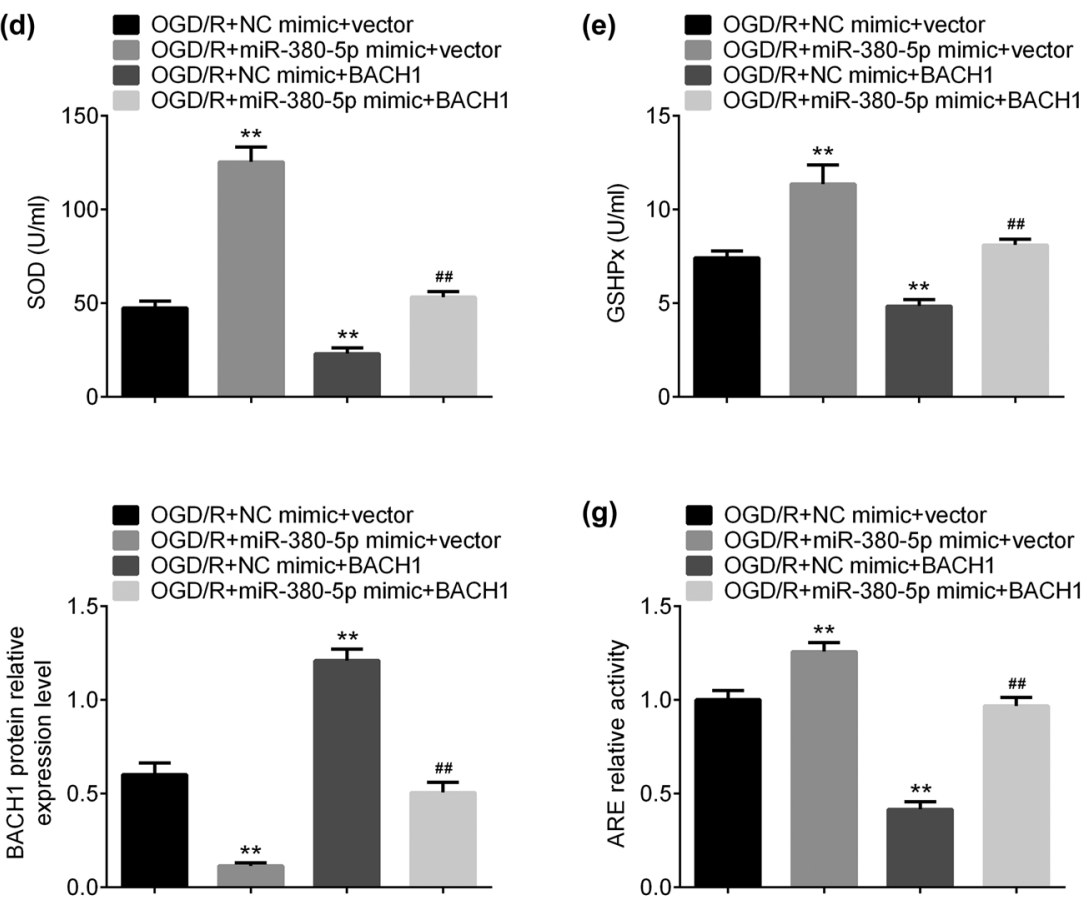

Figure 5: Overexpression of BACH1 reversed miR-380-5p-mediated neuroprotection on SH-SY5Y cells. (a) Overexpression of BACH1 inhibited the increase of cell viability induced by miR-380-5p mimics. (b) Overexpression of BACH1 inhibited the reduction of cell apoptosis induced by miR-380-5p mimics. (c) Overexpression of BACH1 inhibited the reduction of MDA induced by miR-380-5p mimics. (d) Overexpression of BACH1 inhibited the increase of SOD induced by miR-380-5p mimics. (e) Overexpression of BACH1 inhibited the increase of GSHPx induced by miR-380-5p mimics. (f) Overexpression of BACH1 inhibited the downregulation of BACH1 expression induced by miR-380-5p mimics. (g) Overexpression of BACH1 inhibited the increase of ARE activity induced by miR-380-5p mimics. (h) Overexpression of BACH1 inhibited the upregulation of HO-1 and NQO1 mRNA expression induced by miR-380-5p mimics. ${ }^{\star \star} p<0.01 \mathrm{vs} \mathrm{OGD/R}+\mathrm{NC}$ mimic + vector; ${ }^{\# \#} p<0.01$ vs $O G D / R+$ miR-380-5p mimic + vector. 


\subsection{Overexpression of BACH1 reversed miR-380-5p-mediated neuroprotection on SH-SY5Y cells}

Cell viability was increased by miR-380-5p mimics and reduced by overexpression of BACH1 in $\mathrm{SH}-\mathrm{SY} 5 \mathrm{H}$ cells after OGD/R (Figure 5a). Cell apoptosis was reduced by miR-380-5p mimics and induced by overexpression of BACH1 in SH-SY5H cells (Figure 5b). The increase of cell viability and the reduction of cell apoptosis induced by miR-380-5p mimics were inhibited by dual-upregulation of miR-380-5p and BACH1 (Figure $5 a$ and b). MDA level was decreased by miR-380-5p mimics and increased by BACH1 vectors (Figure 5c). miR-380-5p-induced reduction of MDA was prevented by co-transfection of miR-380$5 p$ and BACH1 (Figure 5c). SOD and GSHPx were increased by miR-380-5p mimics and reduced by BACH1 vectors (Figure $5 \mathrm{~d}$ and e). Induction of SOD and GSHPx by miR380-5 mimics was prevented by co-transfection of miR380-5p and BACH1 (Figure $5 \mathrm{~d}$ and e). Protein expression of BACH1 was downregulated by miR-380-5p mimics and upregulated by BACH1 vectors (Figure $5 \mathrm{f}$ ). Inhibition of BACH1 by miR-380-5p mimics was reversed by co-transfection of miR-380-5p and BACH1 (Figure 5f). ARE activity, heme oxygenase-1 (HO-1), and NADPH quinone oxidoreductase 1 (NQO1) mRNA expression were increased by miR-380-5p mimics and decreased by BACH1 vectors (Figure $5 \mathrm{~g}$ and $\mathrm{h}$ ). Dual-upregulation of miR-380-5p and BACH1 inhibited the reduction of ARE activity, HO-1, and NQO1 mRNA expression by miR-380-5p mimics (Figure $5 \mathrm{~g}$ and $\mathrm{h}$ ).

\section{Discussion}

As mentioned in the introduction part, CIR injury could result in serious dysfunction of brain with high risk of disability and mortality and there is no effective treatment for CIR injury [4]. Thus, a better understanding of the mechanism of CIR injury may help to provide the new therapeutic strategy for CIR patients. In this study, expression of miR-380-5p was repressed after OGD/R. Further experiment demonstrated that miR-380-5p could attenuate the neuroblastoma cell death induced by oxidative stress after CIR injury and this process was medicated by direct targeting BACH1 and facilitating NRF2, indicating that miR-380-5p played the neuroprotective role during CIR injury.

Dysregulation of miRNAs has been found in many pathological pathways [11]. Previous study has found that high expression of miR-380-5p promoted cellular survival through target 3'-UTR of p53 in neuroblastoma model, resulting in the poor outcomes of neuroblastoma [15]. Similarly, data of this study have demonstrated that miR-380-5p also contributed to neuroblastoma cell survival after OGD/R. But, in this case, miR-380-5p targeted 3'-UTR of BACH1 to protect brain dysfunction after CIR injury. By heterodimerizing with Maf proteins, BACH1 could inhibit the transcription of HO-1 and NQO1, 2 oxidative stress-response genes, by binding to Maf recognition elements (MAREs) [16]. In this study, both inhibition of miR-380-5p and overexpression of BACH1 have downregulated the mRNA expression of HO-1 and NQO1, in accordance with previous findings. Furthermore, overexpression of BACH1 reversed the neuroprotective effects of miR-380-5p after CIR injury, proving that BACH1 is the direct target of miR-380-5p.

BACH1 and NRF2 are competitors for binding to MAREs in response to oxidative stress [16]. During oxidative stress, NRF2 is activated and releases from Kelch-like ECH-associated protein 1 (Keap1), and translocates into the nucleus, where it competitively binds to MAREs, initiating the transcription of HO-1 and NQO1 [17,18]. Data of this study have showed that expression of NRF2 was upregulated after OGD/R, but was not affected by miR380-5p inhibitors of mimics. However, NRF2 expression in nucleus was increased by miR-380-5p mimic. These data indicated that upregulation of miR-380-5p facilitated the activation of NRF2-Keap1 signaling pathway through repression of BACH1.

After heterodimerizing with MAREs, the target genes of NRF2, called antioxidant response elements (ARE), were also activated [19]. ARE activity was increased by miR-380-5p mimics and decreased by miR-380-5p inhibitors, implying the antioxidant effects of miR-380-5p in neuroblastoma cells after CIR injury. MDA is the terminal production of lipid peroxidation and used to measure the level of lipid peroxidation [20]. As mentioned, SOD and GHSPx are two antioxidant enzymes [7]. Results of this study have manifested that miR-380-5p inhibited MDA level and increased SOD and GHSPx levels, suggesting that miR-380-5p played an antioxidant role in CIR injury, which prevented neuronal cell injury induced by OGD/R.

In conclusion, data of this study have showed that expression of miR-380-5p was repressed after OGD/R. Further experiment demonstrated that miR-380-5p could attenuate the neuroblastoma cell death induced by oxidative stress after CIR injury and this process was medicated by direct targeting BACH1 and facilitating NRF2, indicating that miR-380-5p played the neuroprotective 
role during CIR injury. These results provide the new insight to CIR injury.

Funding information: This research was supported by the Hainan Provincial Natural Science Foundation of China (Funding code: 819QN346), and Suzhou Science and Technology Plan Project (People's Livelihood Science and Technology) (Grant No. SYS2020067).

Author contributions: Yibiao Wang and Min $\mathrm{Xu}$ designed the study and supervised the data collection, Yibiao Wang analyzed and interpreted the data, and Min $\mathrm{Xu}$ prepared the manuscript for publication and reviewed the draft of the manuscript. All authors have read and approved the manuscript.

Conflict of interest: Authors state no conflict of interest.

Data availability statement: All data generated or analyzed during this study are included in this published article.

\section{References}

[1] Hasso AN, Stringer WA, Brown KD. Cerebral ischemia and infarction. Neuroimaging Clin N Am. 1994 Nov;4(4):733-52.

[2] Lapi D, Colantuoni A. Remodeling of cerebral microcirculation after ischemia-reperfusion. J Vasc Res. 2015;52(1):22-31.

[3] Schmidt JM. Heart rate variability for the early detection of delayed cerebral ischemia. J Clin Neurophysiol. 2016 Jun;33(3):268-74.

[4] Ishida A, Kawakami H, Yasuzumi F, Morishita R. Gene therapy for cerebral infarction (cerebral ischemia). No Shinkei. 2002 Mar;54(3):213-9.

[5] Schaller B, Graf R. Cerebral ischemia and reperfusion: the pathophysiologic concept as a basis for clinical therapy. J Cereb Blood Flow Metab. 2004 Apr;24(4):351-71.

[6] Yang J, Chen M, Cao RY, Li Q, Zhu F. The role of circular RNAs in cerebral ischemic diseases: ischemic stroke and cerebral ischemia/reperfusion injury. Adv Exp Med Biol. 2018;1087:309-25.

[7] Chen CH, Hsieh CL. Effect of acupuncture on oxidative stress induced by cerebral ischemia-reperfusion Injury. Antioxid (Basel). 2020 Mar;9(3):248.

[8] Wang Y, Branicky R, Noë A, Hekimi S. Superoxide dismutases: dual roles in controlling ROS damage and regulating ROS signaling. J Cell Biol. 2018 Jun;217(6):1915-28.

[9] Taysi S, Tascan AS, Ugur MG, Demir M. Radicals, oxidative/ nitrosative stress and preeclampsia. Mini Rev Med Chem. 2019;19(3):178-93.

[10] Zhang R, Xu M, Wang Y, Xie F, Zhang G, Qin X. Nrf2-a promising therapeutic target for defensing against oxidative stress in stroke. Mol Neurobiol. 2017 Oct;54(8):6006-17.

[11] Zhang Y, Sun X, Icli B, Feinberg MW. Emerging roles for microRNAs in diabetic microvascular disease: novel targets for therapy. Endocr Rev. 2017 Apr;38(2):145-68.

[12] Ouyang YB, Stary CM, Yang GY, Giffard R. microRNAs: innovative targets for cerebral ischemia and stroke. Curr Drug Targets. 2013 Jan;14(1):90-101.

[13] Lee ST, Chu K, Jung KH, Yoon HJ, Jeon D, Kang KM, et al. MicroRNAs induced during ischemic preconditioning. Stroke. 2010 Aug;41(8):1646-51.

[14] Livak KJ, Schmittgen TD. Analysis of relative gene expression data using real-time quantitative PCR and the 2(-Delta Delta C (T)) method. Methods. $2001 \mathrm{Dec} ; 25(4): 402-8$.

[15] Swarbrick A, Woods SL, Shaw A, Balakrishnan A, Phua Y, Nguyen A, et al. miR-380-5p represses $p 53$ to control cellular survival and is associated with poor outcome in MYCN-amplified neuroblastoma. Nat Med. 2010 Oct;16(10):1134-40.

[16] Zhang X, Guo J, Wei X, Niu C, Jia M, Li Q, et al. Bach1: function, regulation, and involvement in disease. Oxid Med Cell Longev. 2018 Oct;2018:1347969.

[17] Mizumura K, Maruoka S, Shimizu T, Gon Y. Role of Nrf2 in the pathogenesis of respiratory diseases. Respir Investig. 2020 Jan;58(1):28-35.

[18] Igarashi K, Sun J. The heme-Bach1 pathway in the regulation of oxidative stress response and erythroid differentiation. Antioxid Redox Signal. 2006 Jan-Feb;8(1-2):107-18.

[19] Buendia I, Michalska P, Navarro E, Gameiro I, Egea J, León R. Nrf2-ARE pathway: an emerging target against oxidative stress and neuroinflammation in neurodegenerative diseases. Pharmacol Ther. 2016 Jan;157:84-104.

[20] Zhong H, Hao L, Li X, Wang C, Wu X. Anti-inflammatory role of trilobatin on lipopolysaccharide-induced acute lung injury through activation of AMPK/GSK3 $\beta$-Nrf2 pathway. Signa Vitae. 2020 Oct;16(2):160-6. 